\title{
Content-Based Music Genre Classification: Modified AIS-Based Classifier
}

\author{
Noor Azilah Muda ${ }^{1}$, Yun-Huoy $\mathrm{Choo}^{2}$, Norashikin Ahmad ${ }^{3}$ Azah Kamilah Muda ${ }^{4}$ \\ Center for Advanced Computing Technologies (C-ACT) \\ Faculty of Information \& Communication Technology, \\ Universiti Teknikal Malaysia Melaka, \\ 76100 Durian Tunggal Melaka, Malaysia \\ azilah@utem.edu.my ${ }^{1}$; huoy@utem.edu.my²; norashikin@utem.edu.my ${ }^{3}$,azah@utem.edu.my ${ }^{4}$
}

\begin{abstract}
The most important aspect that one should consider in a content-based analysis study is the feature that represents the information. It is common in music analysis study that to identify any song genre, one should know the details that could be used to differentiate the songs. One needs to understand and know which features that can describe the data so that the identifying, labelling, and classification processes will be more precisely done and quicker. This research investigates and analyzes timbre, rhythm, and pitch-based features to classify music genres by extracting low- level features from the songs. The features were extracted from the songs that consist the singer's voice, the instruments and the melody. Two modules of Negative Selection Algorithm; censoring and monitoring are highlighted in this work. We then proposed the Modified AIS-based classification algorithm to solve the music genre classification problem. The results from our experiments demonstrate that the proposed algorithm performs significantly in classifying the music genres.
\end{abstract}

Key words: Artificial Immune System, Negative Selection Algorithm, Censoring and Monitoring, Classification System, Song Genre

\section{INTRODUCTION}

Music analysis has always been an active research work. Major changes can be seen in the music industry resulting from the vast amount of works in this domain. From producing digital songs to distributing them online, or from identifying to retrieving the songs automatically, without technology advancement, these would not be realized. The technology has contributed enormously to the related industry (not just the music but others too for example film making industry). As the process of recognizing the various types of songs genres manually is costly with the huge amount of the songs produced, the manual process has overburdened the music industry.

Imitating human efforts in recognizing the music genres by using advanced technology is a solution to the mentioned scenario. One of the earliest and important works in automating the music genre classification can be found in [1] where the work has emphasized the content analysis of the music.

The basic understanding of the content-based analysis is important before any comprehensive works related to the contents of data can be done. Among the works that are related to the content-based analysis are to discriminate different sounds that come from different sources and analyzing the source contents to identify and recognize the sounds in detail. The process of differentiating audio sounds or classifying them has been a subject of many studies for quite some time now. The audio sounds are represented by descriptive information such as name, file format, sampling rate, or acoustical features like loudness, pitch, or brightness, as briefly mentioned in [2].

Music recognition analysis is a process of automating the tasks performed by humans using recognition methods or techniques. The human recognition process generally is based on a person's auditory skills where a person hears a song and labels it into an appropriate category. During the process, the person will identify the song based on the sounds coming from the singers' vocals and the instruments played.

A song is a combination of singer voices and instruments and these sounds are normally different from one type to another. The rhythm and melody features contained in the musical sounds help the person to differentiate the songs and assign them into different categories. Any person will label the songs based on their hearing sense which has the ability to differentiate and identify the songs. Indirectly, while labelling the songs, humans analyzed the sounds they hear while focusing on the characteristics of the sounds or the features of the music. In automating the recognition process, 
not only the features are important, but an efficient classifier also plays a major role. Machine learning has always been an approach that is investigated, improvised and introduced in music recognition analysis. Another approach like Artificial Immune System (AIS) is also being applied in music analysis area.

Artificial immune system (AIS) is a human-based approach imitating the mechanisms of the immune system in human body. The immune system produces antibodies cells that recognize threats to humans by spotting new and unfamiliar cells. The immune system then categorizes the bad and good organisms, and will only destroy the bad ones. According to [3], AIS is an adaptive mechanism that adapts and imitates the immune technology based on its theories, functions, principles and method to solve problems related to recognizing patterns. According to [4], three key elements that are important to the music genre classification which are recognizing, extracting features and detection the key features.

AIS approach is introduced following algorithms that are modelled to develop artificial aspects of the immune system. Negative Selection Algorithm (NSA) was initially introduced in [5] where the algorithm follows the way the thymus selects the T-cells inside the human body. Its objective is to differentiate the self- and non-self-cells and eliminates unrecognized T-cells in the thymus. [6] provides detailed explanations of how the NSA works. This work proposes a slight modification of the NSA algorithm in classifying music genres. The outcome of the research showed that the Modified AIS-based classifier performances are significantly comparable with other works in the music analysis research area [7].

The Clonal Selection Algorithm (CSA), another algorithm in the AIS domain has also investigated previously to solve pattern recognition problems. IT emphasizes on the mutation process [8]. The CSA has produced good results in solving the problems however, based on our observations, more improvements can be done.

As mentioned previously, the focus of this research is to investigate the low-level features of songs to differentiate the music according to genres. This research is also looking into the NSA with the objective to modify the original algorithm to solve the research problem.

\section{RESEARCH BACKGROUND}

Most of the works related to the music content-based investigation to classify music genres have analyzed the data by looking at low-level music features, for example, analyzing the music frequency, which can lead to analyzing something more detailed and specific like music pitch or music timbre. Works related to the content-based music analysis can be found in the following studies [9], [10]. Other works that are also related to the content-based analysis include: retrieving polyphonic and MIDI types of music [11], features representation studies including metadata standards [12], tools development [13] and symbolic feature sets of the songs [14].

As the music industry is vastly expanding, the technology needed to align with the expansion is also growing parallel with the industry. Using human efforts to identify and classify the various types of a huge amount of songs are costly. Since this has overburdened the industries, providing a solution to automate the human process can be beneficial. Analyzing the music content to provide an intelligent approach to imitate the human process in recognizing the music genre has always been interesting research. The process of recognizing the music mainly analyzes the low-level contents, the characteristics or other features of the music like the artist's voice or instruments played. Among other works of the music analysis are music retrieval, music segmentation, recognition and identification [15].

Author [16] has introduced a labelling process to differentiate categories or genres by utilizing human hearing capability. The results were significant where about 60 percent of the genres were accurately classified. However, using human effort to label the songs is quite costly which makes it impossible and not worth to implement especially if there are a lot of songs that need to be identified. Another related work focusing on similar music contents for music genre classification can be found in [17].

Meanwhile, [18] developed an algorithm that can evolve by adapting itself to the changing environment of the immune system which can also work to cluster and classify. The algorithm discriminates the self or non-self-cells using the cells' size. However, the algorithm can demonstrate the differences between two classes of music genres only.

[19] introduced a classification technique to solve problem then later was modified by [20] to solve the music genre classification problems. The work showed that the modified version classifier which is called the AIRS can now classify more than two music genres. This work significantly better than the previous algorithm as the accuracies recorded are as high as 88 percent. The AIRS has solved not only the two-class music genres only but the cost of employing workers to classify the songs manually as well. The new AIRS emphasizes the clonal rate by increasing the resources assigned to the detectors and introduces the nonlinear coefficient rate. The resources that have lower affinity level were allocated to the detectors parallel during the process. [21], described similar work where the new AIRS classifier proposed a nonlinear method to allocate the resources.

The next section will briefly explain the negative selection algorithm with some background of the immunology system focusing on describing how the immune system works. 


\section{NEGATIVE SELECTION ALGORITHM}

The algorithm was first proposed by a group of researchers to solve problems related to the change detection applications based on the mechanisms of recognising self or non-self-cells. Other works that have applied the algorithm include computer securities [22], computer viruses detection [23], time-series data anomaly detection [25] and network disturbance detection [26].

The negative selection algorithm follows the discrimination of self or non-self-cell processes in the thymus, which is achieved by $\mathrm{T}$-cells having receptors on their surfaces, functioning as a detector to identify the antigens or the foreign proteins. These receptors are generated by a process called the pseudo-random genetic re-arrangement during the generation of T-cells in the thymus [27]. They then go through a censoring process or the negative selective cells task. Inside the thymus, the T-cells that recognise and react to the self-cells are destroyed with the result that only those cells which do not bind themselves to the self-proteins are allowed to leave the thymus. These matured cells circulate in the bloodstream throughout the whole body looking for foreign self-proteins and performing the immunology responses.

The algorithm generally works by eliminating self-cells and randomly transforms the recognised non-self-cells into detectors. These detectors then will recognise a new batch of non-self-cells and eliminate the recognised self-cells. Two modules are involved in the process: censoring and monitoring. Censoring is a process where detectors are randomly generated and monitoring is where the detectors detects non-self-cells. If detected, certain prevention actions are taken. Figure 1 and Figure 2 illustrate the two important stages of the NSA, the censoring and monitoring process.

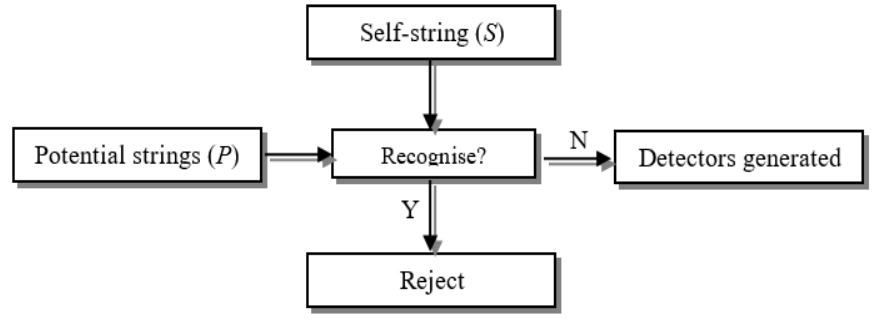

Figure 1: Censoring Stage (detectors are generated randomly)

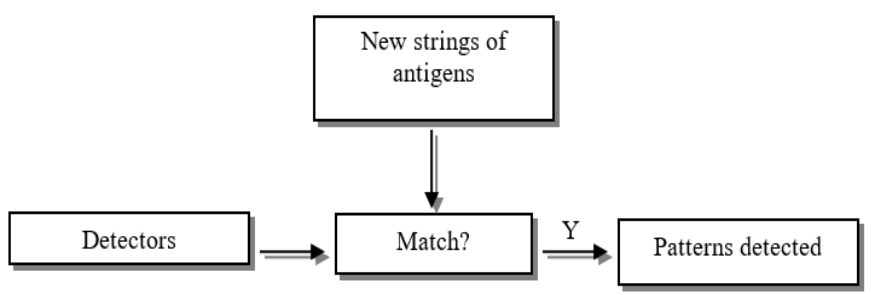

Figure 2: Monitoring Stage (patterns are detected if matched cells occur)
Detectors are very important in the negative selection algorithm, just as they are in the human immune system. It is the main component of the thymus that recognises the cells which are foreign to the human body. From a pattern recognition perspective, the detectors play important roles in recognising the patterns. That is why, researchers are making the effort to study the process of generating the detectors and comparing them in terms of space complexity and time to produce competent detectors.

As the focus of this research is to accurately classify music genres, some modifications were made to the NSA so that it can be used to solve the low performances of previous music genre classification problems. The proposed modified NSA is called the Modified AIS-based music genre classifier. The main problem with the NSA is the mechanism of generating random detectors which can lower the accuracy of classification. The next section will explain the modification that was focusing in generating the detectors mechanism.

\section{MODIFIED AIS-BASED MUSIC GENRE CLASSIFIER}

In any pattern recognition study, the concept idea is to determine which set of features' values belong to a pattern and which set is not. To ensure that the AIS algorithm can be applied to classify the music genre, the mechanism of generating detectors was modified from its original way. Dedicated detectors were produced according to the number of music genres that need to be classified. The modification was done to ensure that the research problem is solved. As the current research is investigating similar patterns in the music genre, the recognition will need precise and dedicated detectors to perform the process [27]. If the assumptions and conclusions of the previous-mentioned works of negative selection algorithm are considered, generating detectors randomly will not guarantee the good performance of the AIS algorithm.

The generated detectors need to be efficient enough so that the detecting process will yield high performance in any identification problem. It is not only focusing on the detectors, but the mechanism to generate the detectors need to be highly effective to generate effective detectors as well. The generalization concept would not be an emphasized criterion as it has been proven that the concept would contribute to the inefficient generated detectors. Censoring and monitoring modules play important roles as they were the fundamental processes of the Modified AIS-based classifier. The detectors are generated exclusively based on how many patterns they should recognize. This is to ensure that there will be a complete set of antibodies that are needed to recognize the antigens. The algorithm includes a process that highlights the XOR-operation in generating the detectors and this process is termed as a detector dedicated generated process. This 
process not only eliminates the random process and the generation process is done in a timely manner, but it can also ensure the generated detectors are appropriate in number for pattern recognition. During the classification process, the detectors are generated from the training data (as this research applies the supervised learning method) which later will be used to test the testing data to find the classification accuracy.

Before any songs can be classified according to genres, the songs feature vectors first need to be transformed into binary strings. At this stage, transformation is very important as the similarity matching techniques that are applied use the binary strings to calculate the classification accuracy based on the song genres. Since the classification is made by recognizing the content of the music, this research will be focusing on analyzing the music contents that are the timbre, pitch and rhythm. The following figure 3 will show the proposed workflow diagram of the proposed classifier.

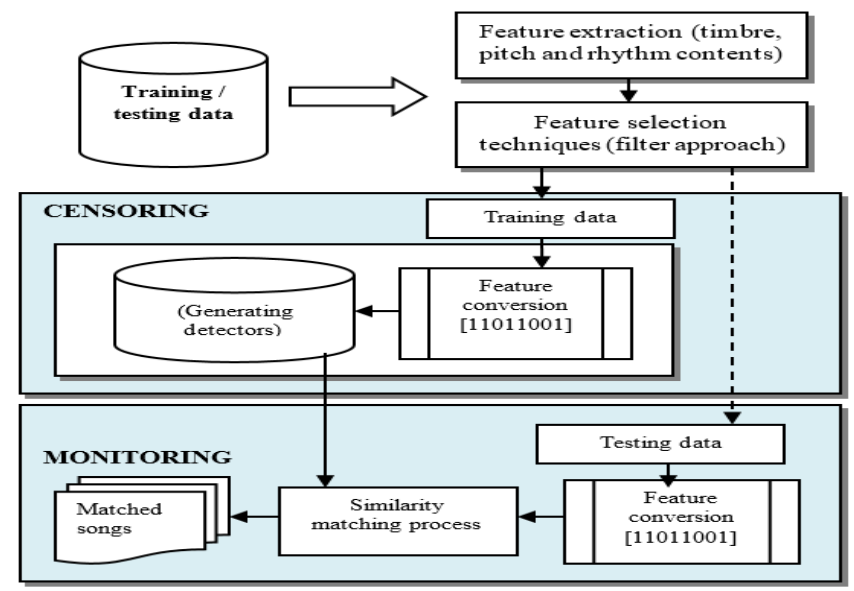

Figure 3: AIS-based music genre workflow diagram

From the workflow diagram, the process begins with the songs feature extraction to get the feature vectors from timbre, rhythm and pitch contents. Then these feature vectors are filtered by a feature selection technique to select only relevant and significant vectors before they are distributed into training and testing data. After the data distribution, these features are then classified and applied to two important aspects of the AIS.

In the censoring process, the song feature vectors are transformed into binary strings where they go through a detector dedicated generation process and are then stored as song detectors. These detectors act as antibodies as they are responsible for identifying similar cells (song antigens). After the censoring process is completed, the testing data is then transformed into binary strings before the dedicated detector generation process is applied and these strings are then stored as songs antigens.

During the monitoring process, the similarity matching process is applied by comparing the song detectors with the song antigens to find out which songs are similar, and those similar songs are identified as matched. The feature vectors are represented using the Hamming mathematical representation because in the similarity matching process, four different binary matching techniques are used to calculate the matched song similarity percentage. The similarity percentage is used to determine whether detectors and antigens are matched by using a threshold value. The following sections are the details of each step in the recognition process.

\subsection{Features Extraction}

By definition, feature extraction is a process to acquire different level song features where it analyses the music content that is useful for recognition process [15]. The extracted features are represented by a numerical mechanism to characterize them. The humans used the information from the songs in the identification process where the hearing sense intelligently select only significant sounds.

Similarly, the automation process follows the hearing sense where first the significant music features are extracted and analysed to recognize the sounds. The human auditory system is efficient and intelligent as it can separate the sounds and categorize the sources. The music contents that provide the features are the pitch (the music pulsation), timbre (differentiate the sounds), and rhythm. Detailed explanations regarding the features extraction process can be found in [29]. The low-level features extracted from these contents contained information that represents different parts of a song. The combination of the features extracted from the music contents will be emphasized in the experimental works to obtain high classification accuracies. Other works that focused on using pitch, rhythm and timbre-related music contents are [30], [31], [32] and [40].

\subsection{Feature Selection}

During the extraction process, some features are irrelevant and redundant. All of these features if they are used in the recognition process will somehow influence the results. To have good outcomes, it is suggested that only related and significant features are used in the recognition process. The reason for keeping the significant and relevant features only is that the selected music features theoretically are significant and can improve the performances and use less time during classification.

To be able to select relevant and significant features only, we used WEKA (developed by a group in the data mining area [33]). WEKA is a well-known tool that is commonly used in machine learning related research. The techniques from the filter approach, the best first and greedy hill-climbing search algorithms were used to select the feature. Once the selection process produces nominated features, the classification 
process will start analyzing those features by applying the modified AIS-based algorithms that will be discussed in the next section.

\subsection{Classification}

Two fundamental modules of the Negative Selection Algorithm (NSA): the censoring and the monitoring modules are the backbones of this step. As explained previously, detectors are created during the censoring process, and comparison between antigens and detectors occurs in the monitoring process. Figure 4 illustrates the modified AIS-based classifier where the conversion process, the censoring and the monitoring modules are emphasized.

Censoring module. This module plays an important part in the proposed classifier as it generates detectors. The generated detectors will determine whether the comparison process will be a success or otherwise. During the process, binary strings is used to represent the features (for example, feature vector -3.4523123 is converted to 101011001). The detectors are created following the number of datasets. The detectors are now labelled as dedicated detectors.

During the process, the comparison between the detectors and the antigens are made to calculate the similarity percentage. The higher the similarities mean the higher the possibilities that both cells are matched. The similarities are determined based on the threshold values where they are used as benchmarks in the process.

As explained previously, both detectors and antigen cells are represented by 15 digits of binaries. During the comparison process to find similarities, each threshold value is set based on the binaries (total binaries for each cell) to calculate the similarity percentage between detectors and antigen (song genres). The experimental works manipulated the threshold values from 1 to 15 (maximum) following the total number of binaries for comparisons. Value " 0 " and "1" is counted to see whether the matched bits exceed the threshold value or lower than the value. As the algorithm generates the detectors from the non-self-cells, the "1" value of the bit is used to detect the non-match antigen-detector cells. The higher the "1" bit from the "0" bit during the comparison mean more non-self-cells are identified. Once found, the cells then will be converted as detectors and are stored for later use in the monitoring module.

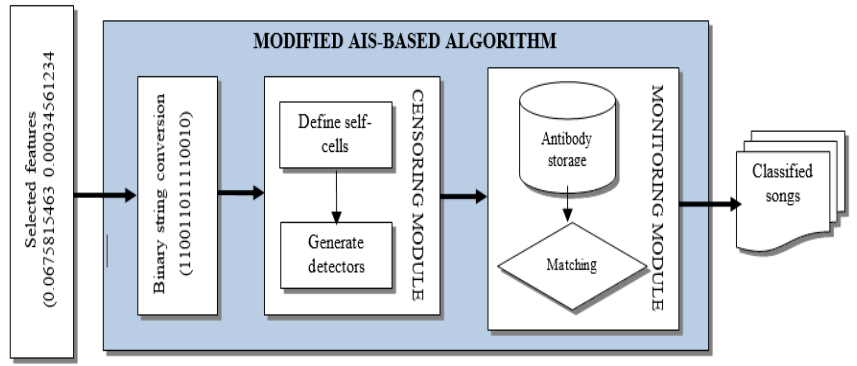

Figure 4: The censoring and monitoring modules
Monitoring module. This module immediately starts after the detectors are generated. During monitoring, similarity comparison is made between detectors and antigens. It is also to calculate the percentage of affinity binding. The calculation is the core part of the classification. Whenever a binary bit " 1 " is produced, the data is considered to bind. However, the word 'match' is used to show similarities between the cells in this research. The emphasized " 1 " is contradicted with the original version of the NSA as it highlighted the "0" to show similarities. The more " 0 " is found, the bigger the similarity between the antigen and the detector.

Once detected as similar and matched, the antigen is considered as self-cell and eliminated. Since the objective of the NSA is to identify non-self-cells, once the 'non-match' cells are detected, the newly detected antigen cell is then considered as threats.

Classification module. All feature vectors from the music contents are combined during classification. Table 1 discusses the classification calculation mechanism. Firstly, the bits' percentage that is matched between antigen and detector cells are identified and calculated. Then, the threshold value percentage where it decides the dataset is matched will be calculated. Lastly, the calculation is done to get the accuracy percentage where all matched dataset is divided by the amount of total tested data.

Table 1: Proposed AIS-based classification method

\begin{tabular}{|l|l|}
\hline \multicolumn{1}{|c|}{ Category } & \multicolumn{1}{c|}{ Calculation formulas } \\
\hline $\begin{array}{l}\text { Data genre } \\
\text { accuracy stage } \\
\text { Threshold }(r) \%\end{array}$ & $\Sigma$ bits_matched / $\Sigma$ features_bits x 100 \\
& $\begin{array}{l}(\Sigma r * \text { num_of_features / } \Sigma \\
\text { bits_per_feature * num_of_features }) \mathrm{x}\end{array}$ \\
& 100 \\
Dataset & (Num_of_genre_match / \\
accuracy stage & num_of_testing_data) x 100
\end{tabular}

\section{EXPERIMENTAL RESULTS}

The work has experimented 1000 Latin songs from 10 different genres. As for performance comparisons, another 1000 of western songs from different categories have also experimented. The performance of the proposed modified AIS algorithm was benchmarked with the R-Chunk, Hamming Distance, Naive Bayes, (full name of J48), and the Sequential Minimal Optimization classifiers using the 2000 songs from Western and Latin groups of songs.

MARSYAS [38] and rhythm pattern extraction tool [39] were used to extract the features from the music contents. For comparison purposes, the training and testing datasets that are ARFF formatted will be experimented with a machine learning classifier and the data file (DAT) format will be tested with the modified AIS-based classifier. 
The classification accuracies from the proposed classifier are then compared with three machine learning classifiers that are the Naives Bayes, J48, and the Sequential Minimal Optimization (SMO) [40]. The machine learning classifiers were applied in the comparison experiments because of they capable to handle complicated pattern recognition problems related to music analysis.

In the experiments, we have prepared training and testing data by percentage and 10 -folds cross-validation data cases. The classification performances are evaluated between the two prepared data sets. The extracted features from the different music contents are combined and the following categories of performances are evaluated:

a) 70/30 percent training/testing data versus the with 10 -folds cross-validation data

b) two different groups of songs, which are the Latin and the Western songs are compared

c) two techniques from the modified AIS-based classifier namely R-Chunk and Hamming Distance were compared with the machine learning classifier (SMO)

The following figures illustrate the classification results according to the setup cases.

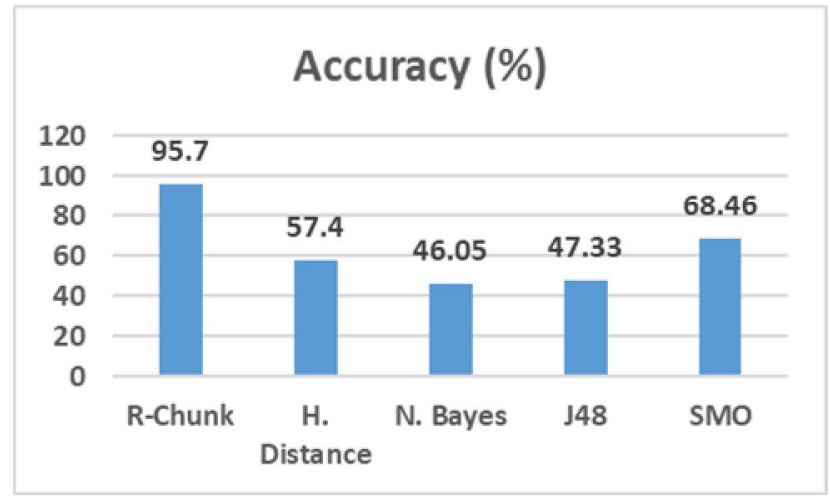

Figure 5: Classification accuracies for all classifiers

From figure 5, the results indicate that the proposed modified AIS-based classifier using the R-Chunk technique has performed better than the rest of classifiers including the Hamming Distance technique that did not perform as well as the R-Chunk technique. However, it still outperformed the Naive Bayes and $\mathrm{J} 48$ classifiers and averagely, the proposed Modified AIS-based classifier has classified the Latin music genres around 76.55 percent which is still higher than the rest of the classifiers. The following figure 6 illustrates the results of the experiments in a different view of comparisons.

From the Latin songs experiments using various classifiers, the R-Chunk technique has significantly classified the Latin music genres almost 30 percent higher than the rest. Compared to the SMO classifier, the Hamming distance technique only managed to accurately classify the Latin music about 10 percent lower than the SMO. This is a bit contradicted with the Western songs experiments as the results showed both techniques were almost equally classifying the music genres. The following Table 3 illustrates the results from both Latin songs and Westerns' using the proposed AIS-based classifiers.

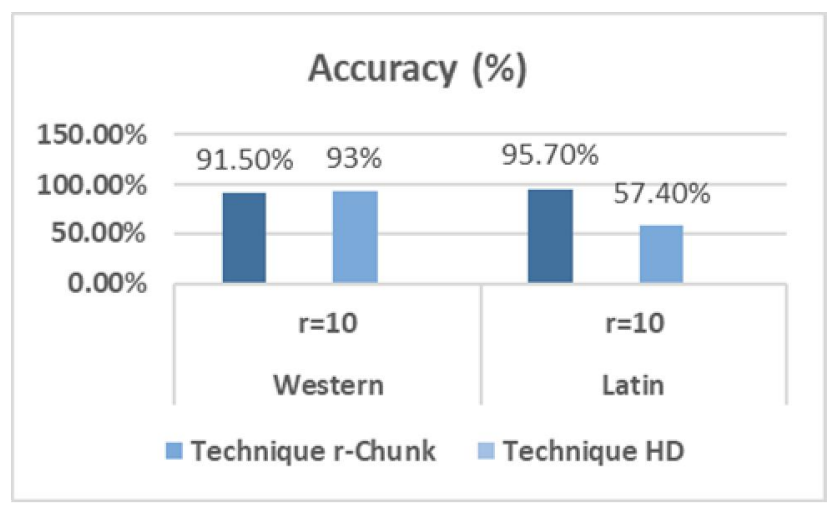

Figure 6: Latin and Western songs comparison

Comparisons between the Latin songs and the Western songs were conducted where the results from applying the modified AIS-based classifier to classify both groups of songs genre were recorded in table 3 . The results showed high classification accuracy in the Western songs experiments however, the accuracy has dropped around 35 percent for the Latin songs. As shown in Figure 6, the slight drop of the Hamming distance performances in the Latin songs was not expected as the Hamming distance (HD) has provided high classification accuracies for other types of ethnicity songs. Assumption of mistakes happen during the data setup where the Latin and the Western songs were stratified into ten-folds for cross-validation purposes is made.

\section{CONCLUSION}

Efforts shown in various music analysis work have proved that these related work has always been an interesting topic and researchers keep contributing to the field in providing solutions, methods and technologies to the industry. As the music industry is not slowing down at all, the studies have produces some breakthroughs which can benefit the music industries and also the customers.

In this paper, we have presented the proposed modified version of algorithm can be applied in music genre classification works. The proposed technique can be regarded as a new alternative technique to solve problems in the music analysis field. From the experimental works, the proposed classifier has outperformed the Naïve Bayes, J48 and SMO classifiers. The experimental works were compared with other works that focused on similar music features and used various classifiers as mentioned previously. 
As the focus of this research is to classify the music genre using the modified AIS-based classifier, the experimental works are conducted using similar low-level features that are extracted from timbre-, pitch- and rhythm-based music contents.

From the classification results, the percentages showed that the proposed classifier has performed well in classifying the music genres. The obtained results are comparable with other previous works where the percentages are not only excellent but they also exceed other works results. From the discussion throughout this paper, this work has presented opportunities to other researchers in this area to fill the gaps, to explore the promising AIS area and to provide solutions to the known and unknown problems that have yet to be discovered.

Future work will be focusing on how to manipulate the threshold values in different ways to increse the performance of the proposed classifier. An exhaustive experiments should be applied to evaluate the significant threshold values that will provide the comparable claasification accuracies.

\section{ACKNOWLEDGEMENT}

This work is funded by Centre of Advanced Computing and Technologies, Faculty of Information and Communication Technology, Universiti Teknikal Malaysia Melaka (UTeM).

\section{REFERENCES}

1. Tzanetakis, G., Ermolinskyi, A. \& Cook, P. Pitch histograms in audio and symbolic music information retrieval. International Society for Music Information Retrieval Conference. Pompidao, Paris, 2002.

2. Liu, C. C. \& Tsai, P. J. A Singer Identification Technique for Content-Based Classification of MP3 Objects. The Tenth International Conference on Information and Knowledge Management, Atlanta, Georgia, USA, 2001.

3. L.N. de Casto and J. Timmis, Artificial immune system: A new computational intelligence approac", Great Britain, Springer, pp. 76-79, 2002.

4. Xiao, R.-B., Wang, L. \& Liu, Y. A framework of AIS based pattern classification and matching for engineering creative design. IEEE First International on Machine Learning and Cybernetics. Beijing, China, 2002.

5. R.-B. Xiao, L. Wang and Y. Liu, A framework of AIS based pattern classification and matching for engineering creative design, in Proceedings of the First International Conference on Machine Learning and Cybernetics, Beijing, China, pp. 1554-1558, 2002.

6. S. Forrest, A.S. Perelson, L. Allen and R. Cherukuri, Self-nonself discrimination in a computer, in Proceedings of 1994 IEEE Computer Society
Symposium on Research in Security and Privacy, Oakland, CA, USA, pp. 202-212, 1994.

7. Muda, N. A. and Wilson, C. and Ling, S. Modified AIS-Based Classifier for Music Genre Classification. In: 11th International Society for Music Information Retrieval Conference, Utrecht, Netherlands, 2010.

8. Golzari, S., Doraisamy, S., Sulaiman, M. N. \& Udzir, N. I. A Hybrid Approach to Traditional Malay Music Genre Classification: Combining Feature Selection and Artificial Immune Recognition System. International Symposium of Information Technology, Vol 1-4, pp. 1068-1073, 2008.

9. Costa, Y.M.G., Olivera, L. S. \& Silla, C. S. An evaluation of Convolutional Neural Networks for music classification using spectrograms. Applied Soft Computing Journal. 52. Pp. 28-38, 2017.

10. Koukoutchos, M., Music Genre Classification. The University of Washington, 2017.

11. Rayar, R., Bennet, M. A., Banu, A. N., Sushanthi, A., \& Rajasekar, M. Music Instrument Sound Classification. IIOAB Journal. Vol.8 (2). 36-41, 2017.

12. Li T., M., O. \& Li, Q. A Comparative Study on Content-Based Music Genre Classification. 26th annual international ACM SIGIR conference on Research and Development in Information Retrieval. Toronto, Canada, 2003.

13. Kim, H. G., Moreau, N. \& Sikora, T. Audio classification based on MPEG-7 spectral basis representations. IEEE Transactions on Circuits and Systems for Video Technology, 2004.

14. Creme, M., Burlin, C., \& Lenain, R., Music Genre Classification. Stanford University, 2016.

15. Picolli, H. C. B. An Evaluation of Symbolic Feature Sets and Their Combination for Music Genre Classification. IEEE International Conference on Systems, Man, and Cybernetics (SMC). Manchester, United Kingdom, 2013.

16. Brecheisen, S., Kriegel, H. P., Kunath, P. \& Pryakhin, A. Hierarchical genre classification for large music collections. 2006 IEEE International Conference on Multimedia and Expo - ICME 2006, Vol 1-5, Proceedings, pp. 1385-1388, 2006.

17. R. Neumayer and A Rauber, Integration of text and audio features for genre classification in music information retrieval, In Proceeding of 29th European Conference on Information Retrieval, Rome, Italy, pp. 724-727, 2007.

18. Sotiropaolos, D. N., Lampropaolos, A. S. \& Tsihrintzis, G. A. Artificial Immune System-Based Music Genre Classification. New Directions in Intelligent Interactive Multimedia, 142, pp. 191-200, 2008.

19. Watkins, A. B. AIRS: A resource limited artificial immune classifier. Computer Science. Mississippi, Mississippi State University, 2001.

20. Hsu, J.-L., Liu, C.-C. \& Chen, A. L. P. Discovering nontrivial repeating patterns in music data. IEEE Transactions on Multimedia, 3, pp. 311-325, 2001. 
21. Liu, H. \& Setiono, R. Feature selection via discretization. IEEE Transactions on Knowledge and Data Engineering, 9, pp. 642-645, 1997.

22. Ayara, M., Timmis, J., Lamos, R. D., Castro, L. N. D. \& Duncan, R. (2002) Negative Selection: How to Generate Detectors in Timmis, J. \& Bently, P. J. (Eds.) International Conference on Artificial Immune System. Canterbury United Kingdom.

23. Forrest, S., Perelson, A. S., Allen, L. \& Cherukuri, R. (1994) Self-nonself discrimination in a computer. IEEE Symposium on Research in Security and Privacy. Oakland, California, USA

24. Dasgupta, D. \& Forrest, S. (1996) Novelty Detection in Time Series Data Using Ideas from Immunology. International Conference on Intelligent Systems. Reno Nevada, USA.

25. Dasgupta, D. (Ed.) (1998) Artifical Immune Systems and Their Applications, Berlin, Germany, Springer.

26. Hofmeyr, S. A. (1999) An immunology of distributed detection and its application to computer security. University of New Mexico.

27. Gonzalez, F., Dasgupta, D. \& Gomez, J. The effect of binary matching rules in negative selection. Genetic and Evolutionary Computation - GECCO 2003. Heidelberg, Springer Berlin, 2003.

28. Muda, N.A., Muda, A.K., Huoy, C.Y., Recognizing music features pattern using modified negative selection algorithm for songs genre classification, Advances in Intelligent Systems and Computing, 2018.

29. Tzanetakis, G. \& Cook, P. (2002) Musical Genre Classification of Audio Signals. IEEE Transactions on Speech and Audio Processing, 10, 293-302.

30. Hartmann, M., Saari, P., Toiviainen, P. \& Lartillot, O. (2013) Comparing Timbre-based Features for Musical Genre Classification. The Sound and Music Computing Conference 2013 (SMC 2013) Stockholm, Sweden.

31. Huang, Y.-F., Lin, S.-M., Wu, H.-Y. \& Li, Y.-S. (2014) Music Genre Classification based on Local Feature Selection Using a Self-Adaptive Harmony Search Algorithm. Data and Knowledge Engineering, 92, 60-76.

32. Chathuranga, D. \& Jayaratne, L. (2013) Automatic Music Genre Classification of Audio Signals with Machine Learning Approaches. GSTF Journal on Computing (JoC), 3.

33. Frank, E., Hall, M., Trigg, L., Holmes, G. \& Witten, I. H. (2004) Data Mining in Bioinformatics using Weka. Bioinformatics, 20, 2479-2481.

34. Draman, A. K. (2009) Authorship Invarianceness for Writer Identification Using Invariant Discretiation and Modified Immune Classifier. University of Technology Malaysia.

35. Balthrop, J., Esponda, F., Forrest, S. \& Glickman, M. (2002) Coverage and Generalisation in an Artificial Immune System. in Langdon, W. B., Cantu-Paz, E., Mathias, K., Roy, R., Davis, D., Poli, R., Balakrishnan,
K., Hanovar, V., Rudolph, G., Wegener, J., Bull, L., Potter, M. A., Schultz, A. C., Miller, J. F., Burke, E. \& Jonaska, N. (Eds.) Conference of Genetic and Evolutionary Computation New York, USA, Morgan Kaufmann Publishers Inc.

36. Gonzalez, F., Dasgupta, D. \& Gomez, J. (2003) The effect of binary matching rules in negative selection. Genetic and Evolutionary Computation - GECCO 2003. Heidelberg, Springer Berlin.

37. Tzanetakis, G. \& Cook, P. MARSYAS: a framework for audio analysis. Organized Sound, 4, pp. 169-175, 1999.

38. Lidy, T. Evaluations of new audio features and their utilization in novel music retrieval applications. Vienna, Vienna University of Technology, 2006.

39. Silla, C. N., Koerich, A. L. \& Kaestner, C. A. A. (2010) Improving automatic music genre classification with hybrid content-based feature vectors. 25th Symposium on Applied Computing. Sierre, Switzerland.

40. Mukkamala S N V Jitendra, Dr Y. Radhika. (2020) A Review: Music Feature Extraction from an Audio Signal. International Journal of Advanced Trends in Computer Science and Engineering., Volume 9 No.2, March -April 2020. 